\title{
Numerical simulation of a quantum particle in a box
}

\author{
Raphaël P Hermann $\dagger$ \\ Institut d'Astrophysique de Cointe, Université de Liège, B-4000 Liège, Belgium \\ Received 6 November 1996, in final form 13 February 1997
}

\begin{abstract}
It is shown how one can get numerical prediction of quantum mechanical particle behaviour without using the Schrödinger equation. The main steps of this development are the non-differentiability hypothesis, the equations of motion entailed by this hypothesis, and the numerical formulation of a simple one-dimensional problem: the particle in a box.
\end{abstract}

\section{Introduction}

Since its development, quantum mechanics has been interpreted in various ways; here we shall start with the Feynman interpretation of quantum mechanics. Feynman and Hibbs [1] described the most important paths of typical quantum mechanical particles as 'quite irregular on a very fine scale'. Abbot and Wise [2] formulated the same observation in a more modern language saying the typical quantum mechanical paths are of fractal dimension two.

More recently Nottale [3,4], relying on an extension of the relativity principle, introduced as a new hypothesis that the typical quantum mechanical paths are nondifferentiable. This led to a radically different description of physics, now embedded in a fractal spacetime. This was the first step of a new theory dubbed scale-relativity, which is now in development. We shall review the main steps of this development, which was presented in [3].

In this paper we shall then show how, by means of numerical simulation, one can get the quantum mechanical behaviour without using the Schrödinger equation. Simply making the non-differentiability hypothesis, not going further in the scale relativistic description, will suffice to achieve this.

\section{Non-differentiability}

We shall first briefly review the main steps entailed by the non-differentiability hypothesis as described in [4]. We assume a continuous non-differentiable space. Considering the geodesics of such a space, we get two straightforward results.

First, knowing that a continuous curve of finite length must be nearly everywhere differentiable (Lebesgue's theorem), we conclude that a nearly nowhere differentiable continuous curve must be of infinite length. More precisely: the length of a geodesic in a non-differentiable space is scale dependent and divergent when the resolution tends to zero.

$\dagger$ E-mail address: r.hermann@.ulg.ac.be 


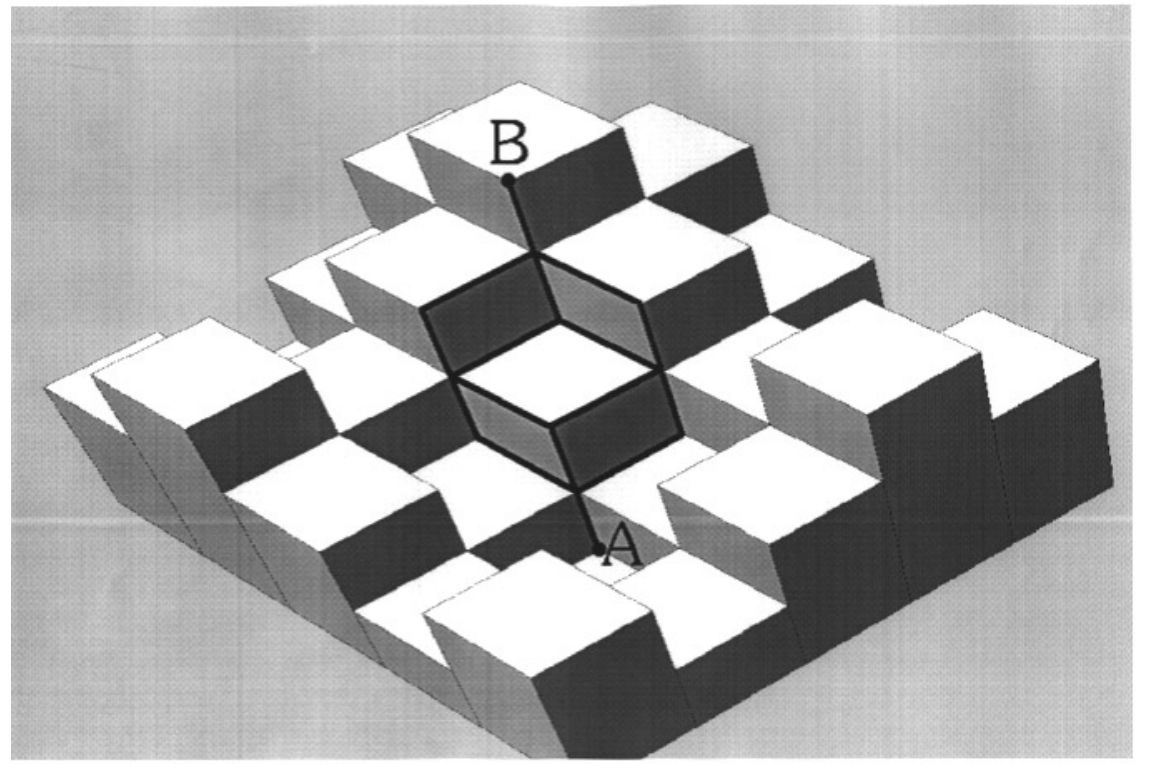

Figure 1. Fractal space. The bold lines show the eight geodesics between A and B. The space being fractal, this is repeated at all resolutions, so the number of geodesics is infinite.

Second, in a non-differentiable space there are an infinity of geodesics between any two points. This will not be proved here but figure 1 shows that even in a simple orthogonal fractal space this statement holds.

We describe the position vector of a particle by a finite, continuous fractal 3 -function $\boldsymbol{x}(t, \mathrm{~d} t)$ explicitly dependent on the time resolution. On our infinite set of geodesics we may now write an infinitesimal element of motion:

$$
\mathrm{d} X_{i}=\mathrm{d} x_{i}+\mathrm{d} \xi_{i}
$$

where $\mathrm{d} x_{i}=\left\langle\mathrm{d} X_{i}\right\rangle$, which entails that $\left\langle\mathrm{d} \xi_{i}\right\rangle=0(i=1,2,3$ and represents the $x, y, z$ directions; $\mathrm{d} \xi$ should depend on an extra index $v$ referring to the various geodesics, which is omitted). The symbol \langle\rangle stands for an average over the omitted index $\nu$. $d \xi_{i}$ is the difference between the position vector $\mathrm{d} X_{i}$ (light dashed line of figure 2) on an actually considered geodesic (bold dashed line of figure 2) and the mean position vector $\mathrm{d} x_{i}$ (bold line of figure 2) on the set of geodesics.

Non-differentiability entails that

$$
\lim _{\mathrm{d} t \rightarrow 0^{+}} \frac{\boldsymbol{x}(t+\mathrm{d} t)-\boldsymbol{x}(t)}{\mathrm{d} t} \neq \lim _{\mathrm{d} t \rightarrow 0^{+}} \frac{\boldsymbol{x}(t)-\boldsymbol{x}(t-\mathrm{d} t)}{\mathrm{d} t}
$$

for nearly all points on every particular geodesic considered. Following Nelson [5], we then define mean forward and backward derivatives:

$$
\frac{\mathrm{d}_{+}}{\mathrm{d} t} \boldsymbol{y}(t)=\lim _{\mathrm{d} t \rightarrow 0^{+}}\left\langle\frac{\boldsymbol{y}(t+\mathrm{d} t)-\boldsymbol{y}(t)}{\mathrm{d} t}\right\rangle \quad \frac{\mathrm{d}_{-}}{\mathrm{d} t} \boldsymbol{y}(t)=\lim _{\mathrm{d} t \rightarrow 0^{+}}\left\langle\frac{\boldsymbol{y}(t)-\boldsymbol{y}(t-\mathrm{d} t)}{\mathrm{d} t}\right\rangle
$$

(the existence of these limits is discussed in [3, p 144]) which we apply to the position vector, obtaining

$$
\frac{\mathrm{d}_{+}}{\mathrm{d} t} \boldsymbol{x}(t)=\boldsymbol{b}_{+} \quad \frac{\mathrm{d}_{-}}{\mathrm{d} t} \boldsymbol{x}(t)=\boldsymbol{b}_{-} .
$$




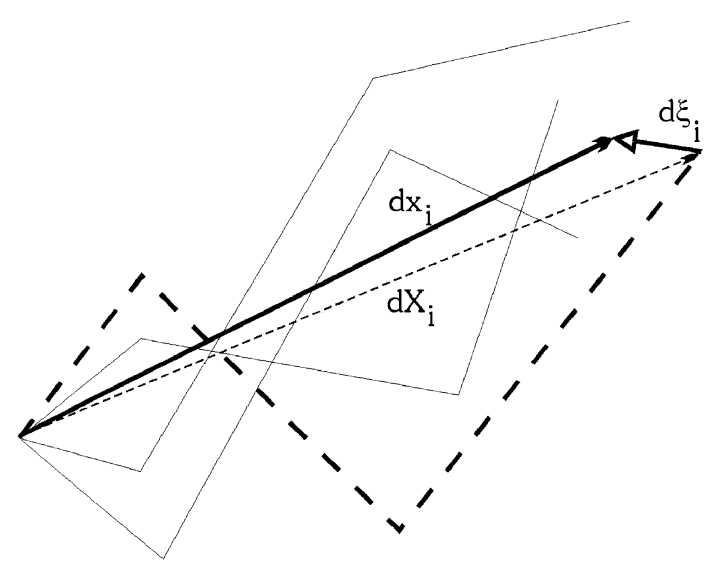

Figure 2. Set of geodesics and average.

Putting (1) and (3) together, we get

$$
\mathrm{d} \boldsymbol{x}(t)=\boldsymbol{b}_{+}[\boldsymbol{x}(t)] \cdot \mathrm{d} t+\mathrm{d} \xi_{+}(t)=\boldsymbol{b}_{-}[\boldsymbol{x}(t)] \cdot \mathrm{d} t+\mathrm{d} \xi_{-}(t)
$$

which describes the position vector as a stochastic process. Note that at a given point the information has been doubled: the two processes are to be taken in account. We assume that (5) is a Wiener process [6], this means that the $d \xi$ 's are supposed to have a mean of zero (true by construction), to be Gaussian, mutually independent and such that

$$
\begin{aligned}
& \left\langle\mathrm{d} \xi_{+i}(t) \cdot \mathrm{d} \xi_{+j}(t)\right\rangle=2 \mathcal{D} \delta_{i j} \mathrm{~d} t \\
& \left\langle\mathrm{~d} \xi_{-i}(t) \cdot \mathrm{d} \xi_{-j}(t)\right\rangle=-2 \mathcal{D} \delta_{i j} \mathrm{~d} t
\end{aligned}
$$

where $\mathcal{D}$ is a constant (dimensionally a diffusion coefficient); the - sign for the second equation is necessary because $\mathrm{d} t<0$ in that equation. The $\mathrm{d} t$ dependence is a consequence of the fractal dimension two of Feynman, and Abbot and Wise (further explanation can be found in [3]).

The only real assumptions are the Gaussian character of the process, but this is not essential to obtain our results (this will be shown in section 3), and the mutual independence (i.e. the presence of the $\delta_{i j}$ Kronecker symbol). The last could perhaps be relaxed, but this is beyond the scope of this paper.

\section{Equation of motion}

Starting from a generic function $f(\boldsymbol{x}, t)$, we expand it in right and left Taylor series to second order and we then replace the $\mathrm{d} x_{i}$ 's with the sum $\mathrm{d} X_{i}=\mathrm{d} x_{i}+\mathrm{d} \xi_{i}$. Averaging over the set of geodesics, we obtain ([7 p 1081]):

$$
\begin{aligned}
& \frac{\mathrm{d}_{+} f}{\mathrm{~d} t}=\left(\frac{\partial}{\partial t}+\boldsymbol{b}_{+} \cdot \nabla+\mathcal{D} \Delta\right) f \\
& \frac{\mathrm{d}_{-} f}{\mathrm{~d} t}=\left(\frac{\partial}{\partial t}+b_{-} \cdot \nabla-\mathcal{D} \Delta\right) f .
\end{aligned}
$$

The development of (7) shows that the Gaussian hypothesis for the $\mathrm{d} \xi_{i}$ 's is not essential, in fact the Laplacian operator is a consequence of the fractal dimension two alone. Let us 
now define two new mean velocities:

$$
\boldsymbol{V}=\frac{1}{2}\left(\boldsymbol{b}_{+}+\boldsymbol{b}_{-}\right) \quad \boldsymbol{U}=\frac{1}{2}\left(\boldsymbol{b}_{+}-\boldsymbol{b}_{-}\right) .
$$

We can combine them in a single complex velocity:

$$
\mathcal{V}=\boldsymbol{V}-\mathrm{i} \boldsymbol{U} .
$$

By the same way we define two new derivatives:

$$
\frac{\mathrm{d}_{V}}{\mathrm{~d} t}=\frac{1}{2} \frac{\mathrm{d}_{+}+\mathrm{d}_{-}}{\mathrm{d} t} \quad \frac{\mathrm{d}_{U}}{\mathrm{~d} t}=\frac{1}{2} \frac{\mathrm{d}_{+}-\mathrm{d}_{-}}{\mathrm{d} t}
$$

which we also combine into a single complex derivative:

$$
\frac{d}{\mathrm{~d} t}=\frac{\mathrm{d}_{V}}{\mathrm{~d} t}-\mathrm{i} \frac{\mathrm{d}_{U}}{\mathrm{~d} t}=\left(\frac{\partial}{\partial t}-\mathrm{i} \mathcal{D} \Delta+\mathcal{V} \cdot \nabla\right) .
$$

Here, Nottale makes the fundamental hypothesis that 'the passage from classical mechanics to the new non-differentiable mechanics can be implemented by a unique prescription: Replace the standard time derivative with the new complex derivative'. ([3, p 148].)

\section{Solution of the equation of motion}

Nottale at this point shows the equivalence of non-differentiable mechanics and quantum mechanics (QM) by developing the mathematical formalism, starting with Newton's equation and finally obtaining the Schrödinger equation.

We shall show here that, from this low-level description we have now reached, it is possible to simulate some simple QM problems.

Let us start with our complex Newton equation:

$$
-\nabla \mathcal{U}=m \frac{d}{\mathrm{~d} t} \mathcal{V} .
$$

Since $\mathcal{U}$, being a potential, is a real quantity, we separate (12) into real and imaginary parts:

$$
\begin{aligned}
& m\left(\frac{\partial}{\partial t} \boldsymbol{V}-\mathcal{D} \Delta \boldsymbol{U}+(\boldsymbol{V} \cdot \nabla) \boldsymbol{V}-(\boldsymbol{U} \cdot \nabla) \boldsymbol{U}\right)=-\nabla \mathcal{U} \\
& m\left(\frac{\partial}{\partial t} \boldsymbol{U}+\mathcal{D} \Delta \boldsymbol{V}+(\boldsymbol{V} \cdot \nabla) \boldsymbol{U}+(\boldsymbol{U} \cdot \nabla) \boldsymbol{V}\right)=0 .
\end{aligned}
$$

We shall restrict ourselves here to the simplest solution of this system: a one-dimensional problem with infinite limit conditions and without force (thus $\mathcal{U}=$ constant), i.e. a particle in a box. The average classical velocity of such a particle is expected to be zero; and $\boldsymbol{V}$, being the sum of the forward and backward velocity, is expected to be this classical velocity. So our system reduces to the one-dimensional one:

$$
\begin{aligned}
& -\mathcal{D} \Delta U-(U \cdot \nabla) U=0 \\
& \frac{\partial}{\partial t} U=0 .
\end{aligned}
$$

The second equation indicates that $U$ is a function of $x$ alone, so we finally obtain

$$
\frac{\partial}{\partial x}\left(\frac{\partial}{\partial x} \mathcal{D} \cdot U(x)+\frac{1}{2} U^{2}(x)\right)=0 .
$$

This can be solved,

$$
U(x)=\sqrt{2 k_{1}} \operatorname{tg}\left(\frac{-\sqrt{2 k_{1}} x}{2 \mathcal{D}}+k_{2}\right)
$$


where the limit conditions will determine the integration constants $k_{1}$ and $k_{2}$.

$U$ being a difference of velocities, we interpret it as a kind of acceleration [3]. We can thus reasonably suppose that $U \rightarrow+\infty$ on the left border (that is $x \rightarrow 0$ ) and $U \rightarrow-\infty$ on the right border (that is, conventionally $x \rightarrow a$, if our 'box' is of size $a$ ).

The limit conditions yield

$$
U(x)=\frac{2 \mathcal{D} n \pi}{a} \operatorname{tg}\left(-\frac{n \pi x}{a}+\frac{\pi}{2}\right) .
$$

Here we remember that (using (5), (8) and $V=0$ )

$$
\mathrm{d} x(t)=U(x(t)) \cdot \mathrm{d} t+\mathrm{d} \xi_{+}(t)=-U(x(t)) \cdot \mathrm{d} t+\mathrm{d} \xi_{-}(t) .
$$

We can choose one of these equations (describing respectively the behaviour of the particle 'towards' and 'away from' a point) to simulate the trajectory of our particle.

Furthermore, it has been shown by Pissondes [8], that when there are no external forces applied on the particle the energy expression becomes

$$
T=\frac{m}{2} \mathcal{V}^{2}-\mathrm{i} m \mathcal{D} \nabla \mathcal{V}
$$

This is precisely the expression differentiated in (15) multiplied by $m$. So, in our case we have

$$
T=k_{1} m=\frac{2 n^{2} \pi^{2} \mathcal{D}^{2}}{a^{2}}
$$

which is exactly the quantum energy $\left(E=n^{2} \pi^{2} \hbar^{2} / 2 m a^{2}\right)$ (after the substitution $\mathcal{D} \rightarrow$ $\hbar / 2 m)$. This replacement is precisely the condition necessary to obtain the Schrödinger equation from (11) (this is shown in [3]).

\section{Numerical simulation}

The stochastic process we have to consider now explicitly becomes

$$
\mathrm{d} x(t)=\frac{2 \mathcal{D} n \pi}{a} \operatorname{tg}\left(-\frac{n \pi x}{a}+\frac{\pi}{2}\right) \cdot \mathrm{d} t+\mathrm{d} \xi_{+}(t)
$$

where $\mathrm{d} \xi_{+}(t)$ is a random variable of a Gaussian distribution and is of width $\sqrt{2 \mathcal{D} \mathrm{d} t}$. We simplify all expressions by taking $2 \mathcal{D} \cdot \mathrm{d} t=1$, so our process can finally be written as

$$
\mathrm{d} x(t)=\frac{n \pi}{a} \operatorname{tg}\left(-\frac{n \pi x}{a}+\frac{\pi}{2}\right)+N(0,1)
$$

where $N(0,1)$ stands for the normalized random variable.

We cannot expect a numerical simulation of this process to give us directly the trajectory of a particle, since we know of the huge ratio between quantum timescales and classical timescales. In fact we would have to memorize about $10^{8}$ positions. However, we shall show a piece of such a trajectory in figure 7 later. The output of this simulation will thus be a profile of the density of the presence of the particle in the box, $r(x)$. To construct it we divide our box into 600 pieces and count the number of timesteps the particle is in each specific box.

On our schemes, the $x$ position in the box is drawn horizontally, and the number of occurrences vertically. So a point of our curves has to be understood as $(x, y) \Leftrightarrow$ (box number $x$, amount of steps for which the particle was in box $x$ ). The results are always normalized. The continuous curves give the result of our simulation. The dashed curves are the result of conventional quantum mechanics, with the same normalization as the numerical result $\left(r_{\mathrm{QM}}(x)=\sin ^{2}(n \pi x / a)\right)$ [9]. 


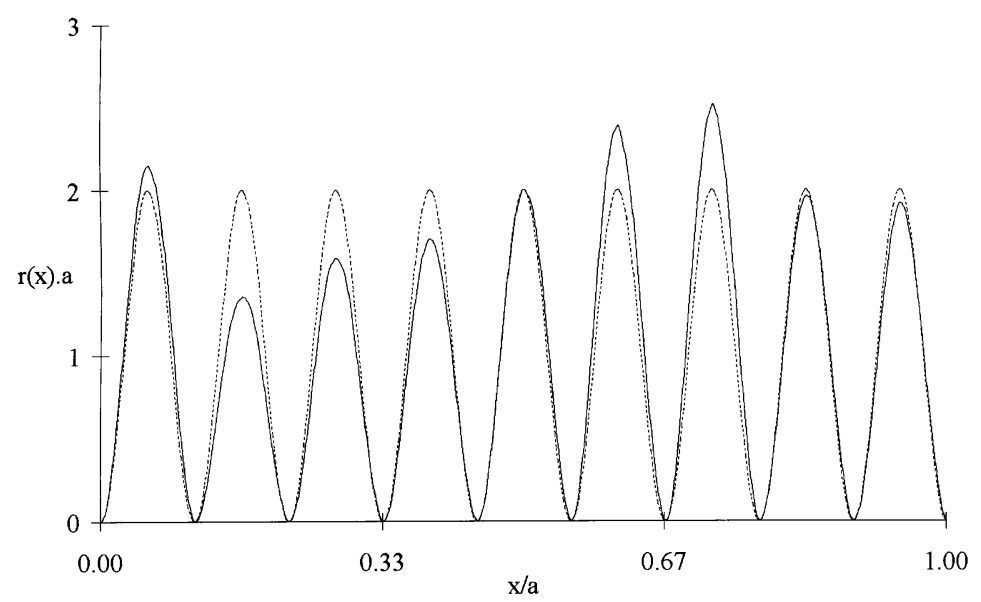

Figure 3. Result with $(n=9)$.

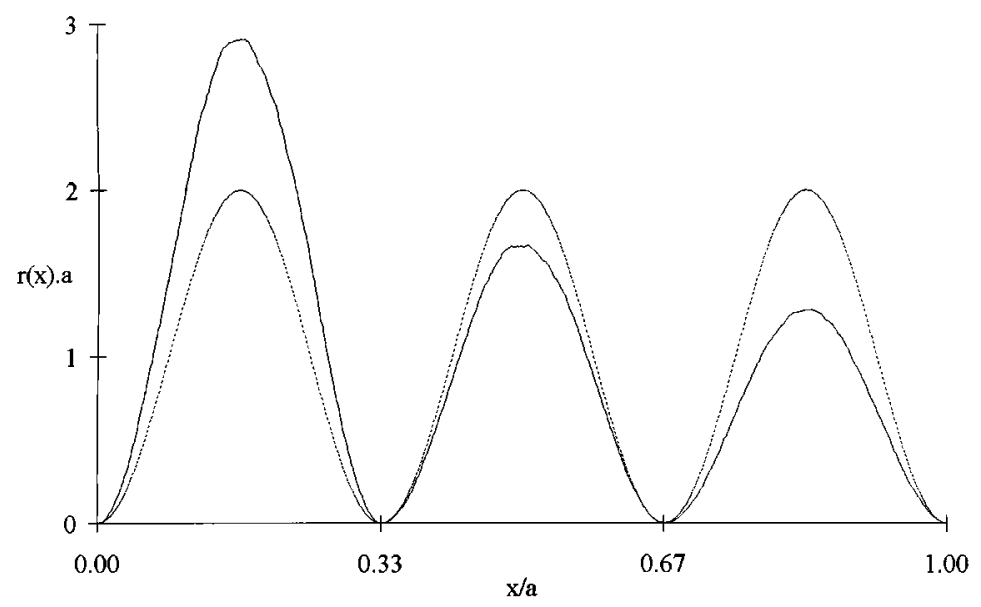

Figure 4. Result with $(n=3)$.

Figures 3 and 4 show a first attempt of modelization with $n=9\left(10^{8}\right.$ steps $)$ and $n=3$ $\left(5 \times 10^{8}\right.$ steps), respectively. As we can see in figure 5 which is a magnification of the right peak of figure 4 , the individual peak shows an excellent agreement with the theoretical $\sin (x)^{2}$ profile normalized for that peak (dashed curve) [9]. The problem of exchange between the different peaks seems less satisfactory.

There are two possibilities to improve on our result: first, by using more steps in time, which is not machine-time friendly; second, by using the arbitrary initial conditions to our advantage. In fact, when we start the simulation we have to put our particle in a specific point of the box. What we propose here is to restart our problem after, say, $10^{5}$ steps in time with a new starting position. This leads to a better 'thermalization' of the system and increases convergence [10]. In fact this is equivalent to the simultaneous simulation of multiple particles with different initial positions (which could be treated vectorially). 


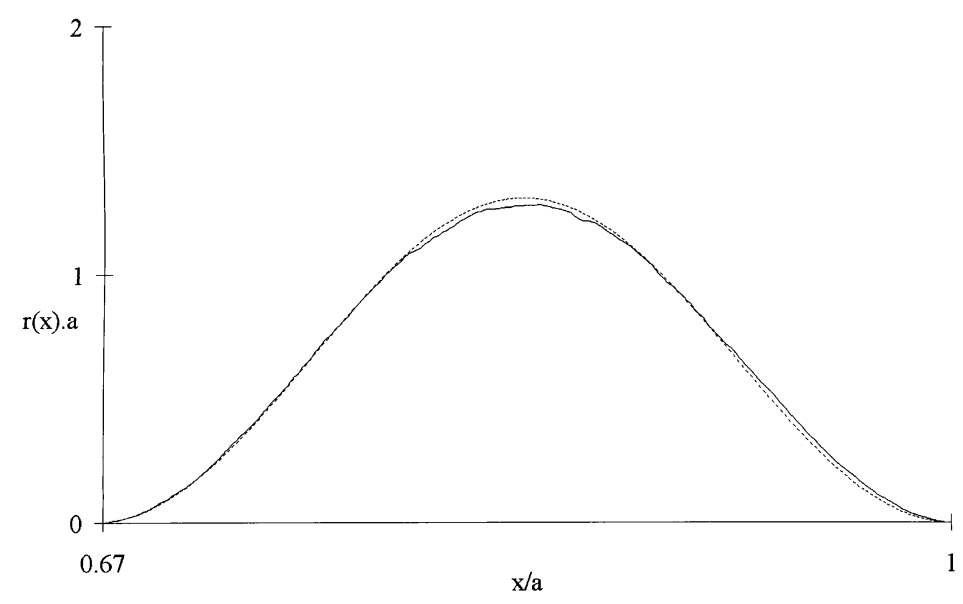

Figure 5. Magnification of the right peak of figure 4 .

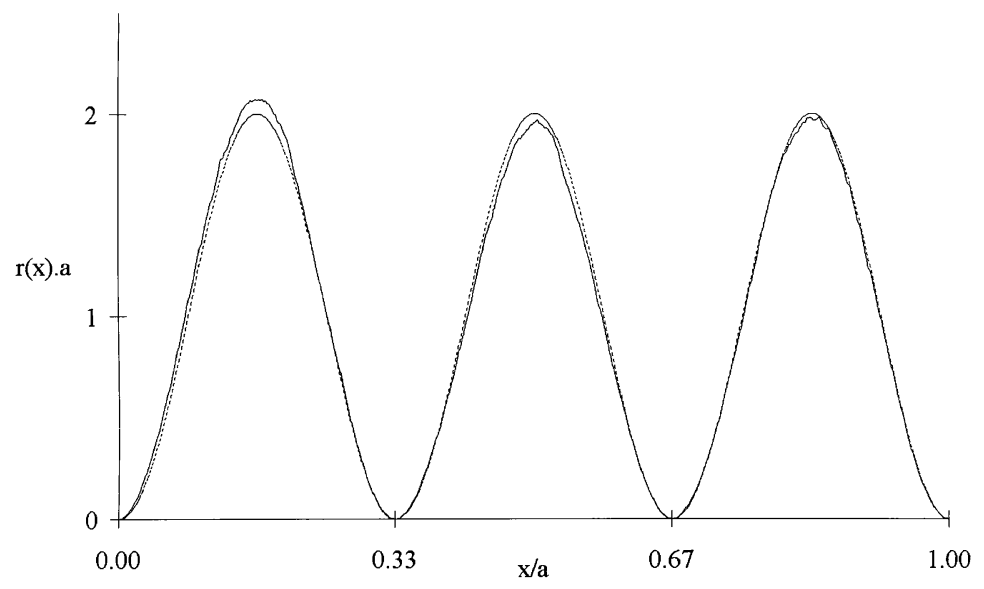

Figure 6. Corrected simulation with $(n=3)$.

Figure 6 shows the result of such a 'pseudo-multitrajectory' simulation, with $n=3$ and $10^{8}$ steps. If we compare with figure 4 , we see the dramatic increase of convergence. The fit between the quantum mechanical result and our simulation (data of figure 6) is now excellent:

$$
\begin{aligned}
& \sigma=\sqrt{\frac{\sum_{i=1}^{600}\left(r_{\mathrm{QM}}(i)-r(i)\right)^{2}}{600}}=0.041 \\
& \rho=\frac{\sum_{i=1}^{600}\left(r_{\mathrm{QM}}(i)-\left\langle r_{\mathrm{QM}}\right\rangle\right)(r(i)-\langle r\rangle)}{\sum_{i=1}^{600}\left(r_{\mathrm{QM}}(i)-\left\langle r_{\mathrm{QM}}\right\rangle\right)^{2} \sum_{i=1}^{600}(r(i)-\langle r\rangle)^{2}}=0.9983
\end{aligned}
$$

where $\sigma$ is the standard deviation and $\rho$ is the correlation coefficient between the quantum mechanical result and our result ( \langle\rangle stands for the average). 


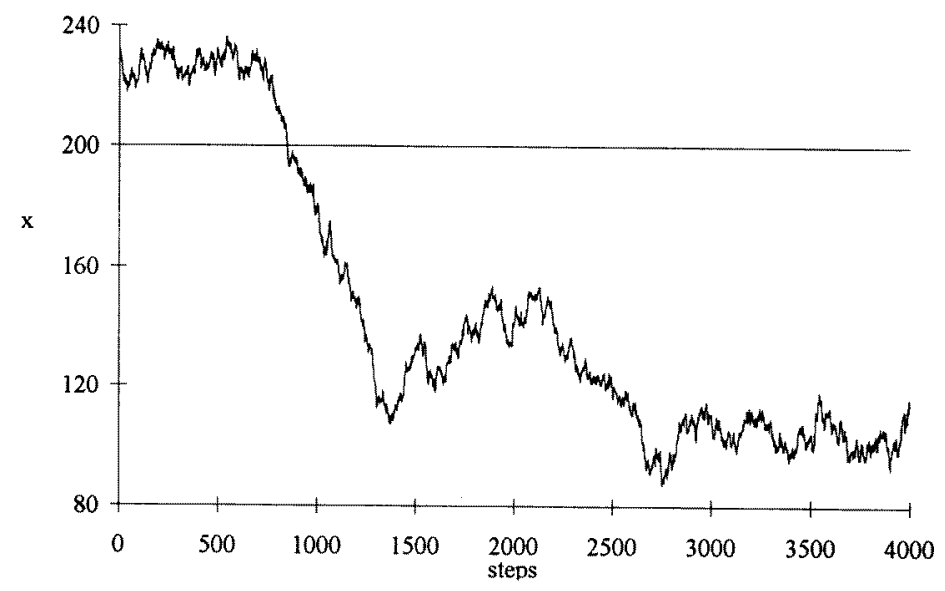

Figure 7. Trajectory of a particle around a density node.

Figure 7 shows a fractal trajectory produced by our simulation around a node (horizontal line), for 4000 steps in time (horizontally). In this example $n=3$ and $a=600$ (node at $x=a / 3=200)$.

Some comments should be considered when speaking about 'quantum trajectories'. The concept of quantum trajectory is not incompatible with the traditional view of QM: the fact that we obtained our results illustrates this. One should also remember that the trajectories mentioned are continuous. The quantum 'jumps' between two peaks occur continuously as shown in figure 7, which in fact is an illustration of such a 'jump' from the central peak to the left peak of figure 6 .

\section{Conclusion}

We have shown that a quantitative correct prediction of the behaviour of a quantum particle in a box can be obtained without writing explicitly the Schrödinger equation nor using any conventional quantum axiom. In fact, it has been shown that the assumption of nondifferentiability of space alone is suffice to obtain this result (being in the domain of nonrelativistic QM - the relativistic extension will require a non-differentiable time as well).

A more detailed discussion about this equivalence of non-differentiability and the Schrödinger equation can be found in $[3,11]$, were it is in fact shown that equation (12) is equivalent to Schrödinger's equation, which can be seen as a 'prime integral' of (12).

An extension to other problems such as the harmonic potential has shown (in preparation) that this method remains valid. Furthermore, a detailed study of the influence of the fractal dimension and of the time resolution is expected to yield results about the understanding of the classical-quantum transition [10] (as related to the fractal dimension transition of the trajectories from one to two).

\section{Acknowledgments}

I especially thank L Nottale and J Perdang for many helpful discussions, and A Lejeune for providing computing facilities. 


\section{References}

[1] Feynman R P and Hibbs A R 1965 Quantum Mechanics and Path Integrals (New York: McGraw-Hill)

[2] Abbot L F and Wise M B 1981 Dimension of a quantum mechanical path Am. J. Phys. 49 37-9

[3] Nottale L 1993 Fractal Space-Time and Microphysics, Towards a Theory of Scale Relativity (Singapore: World Scientific)

[4] Nottale L 1989 Fractals and the quantum theory of spacetime Int. J. Mod. Phys. A 4 5093-4

[5] Nelson E 1985 Quantum Fluctuations (Princeton, NJ: Princeton University Press)

[6] Welsh D J A 1970 Mathematics Applied to Physics (New York: Springer) p 465

[7] Nelson E 1966 Derivation of Schrödinger equation from Newtonian mechanics Phys. Rev. 150 1079-85

[8] Pissondes J-C 1996 Commun. Math. Phys. submitted

[9] Cohen-Tannoudji C, Diu B and Laloe F 1973 Mécanique Quantique (Paris: Hermann)

[10] Hermann R 1995 Le concept de temps en Physique (Université de Liège: Mémoire de licence) pp 92-128

[11] Nottale L 1995 Scale relativity: from quantum mechanics to chaotic dynamics Chaos Solitons Fractals 6 399-410 